\title{
Experimental and numerical tests of thermo-mechanical processes occurring on brake pad lining surfaces
}

\author{
P. Baranowski ${ }^{1}$, K. Damziak ${ }^{1}$, J. Malachowski $^{1}$, L. Mazurkiewicz ${ }^{1}$, \\ M. Kastek ${ }^{2}$, T. Piatkowski ${ }^{2}$, H. Polakowski ${ }^{2}$ \\ ${ }^{1}$ Department of Mechanics and Applied Computer Science, \\ Military University of Technology, Warsaw, Poland \\ ${ }^{2}$ Institute of Optoelectronics, Military University of Technology, \\ Warsaw, Poland
}

\begin{abstract}
One of the most important systems in cars and trucks are brakes. The braking temperature on a lining surface can rise above $500^{\circ} \mathrm{C}$. This shows how lining requirements are so strict and, what is more, continuously rising. Besides experimental tests, a very supportive method for investigating processes which occur on the brake pad linings are numerical analyses. This paper presents the results of laboratory brake lining tests in the special test rig, called IL-68. Furthermore, based on this testing machine, the numerical model was developed. In the final stage computations done using a dynamic code called LS-Dyna were compared with laboratory tests.
\end{abstract}

Keywords: numerical simulations, brake systems, thermo-mechanical coupling, thermovision, infrared measurements.

\section{Introduction}

Typically, braking from nominal speed to full stop, takes about 5-15s. At that time, most of the kinetic energy of a vehicle is changed into heat generated in the brake system [1]. Heat emitted at the working surfaces of a brake is causing heat stroke directed into the components of friction parts. Imperfections of the surface, which may be associated with shape errors and geometric structure, causes uneven temperature distribution, where local maxima may exceed the 
melting temperature of the lining. Geometric structure of the surface includes roughness, waviness and surface defects. Thermal phenomena in the pair brake pad - disc, is particularly important in special vehicles, both civilian and military. Their large masses or high velocities require higher braking forces. Due to permanent deformation in the contact area, increasing load on the direction normal to the contact surfaces, this causes an increase of actual contact surface, up to the maximum value for a given friction part [2]. This leads to a generation of a large amount of heat. Operation in extreme conditions, such as in mountains, with frequent braking, can lead to overheating and - in extreme cases - to the destruction of a brake system [3].

The key issue for an engineer designing a new brake is to measure or to predict both average and local temperatures which are a key factor in estimation of the brake lifetime and effectiveness. In this present paper, temperatures obtained from experimental and numerical tests are studied.

\section{Special test rig IL-68 overview}

Experimental tests were conducted in the Institute of Aviation in Warsaw on the test machine called IL-68, which major elements are shown on the picture below. The main component of IL-68 is the so-called frictional unit, shown in Figure 1, consisting of:

- rotational head, which convey a shaft torque and where counter samples are placed,

- translational head, where samples of coatings are placed and pressed against counter samples.

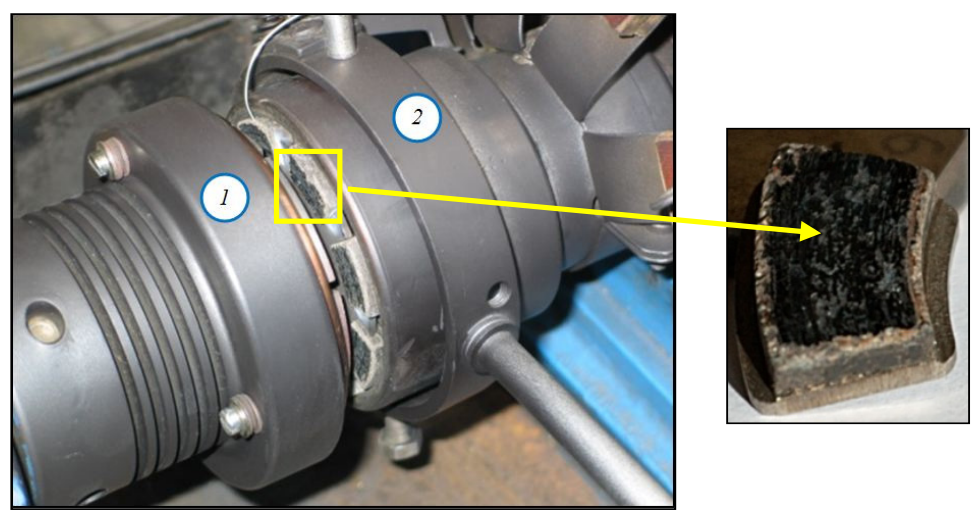

Figure 1: $\quad$ Frictional unit of the IL-68 rig: rotational head (1), translational head (2).

The stand allows for examination of frictional pairs without the need of building a full-scale prototype of a brake. Choosing adequately the geometry of tested samples, it is possible to simulate the brake performance, using relatively small objects. The sample size was approximately $16 \times 10 \times 4 \mathrm{~mm}(\mathrm{~W} \times \mathrm{H} \times \mathrm{D})$, 
outer diameter of $72 \mathrm{~mm}$. Variable rotational speeds of shaft, in the range 0$9000 \mathrm{rpm}$, allows users to simulate a wide range of linear speed in the friction pair. In the described test, it was set at $6000 \mathrm{rpm}$. The moment of inertia of the rotating masses which simulate the energy dissipation similar to the energy dissipated in the real brake, can be set within the limits of $0.154-1.54 \mathrm{~kg} \cdot \mathrm{m}^{2}$ and $0.098 \mathrm{~kg} \cdot \mathrm{m}^{2}\left(0.412 \mathrm{~kg} \cdot \mathrm{m}^{2}\right.$ during tests). In addition, the force acting on a friction pair can be adjusted from 0 to $5.88 \mathrm{kN}$. The stand allows for measuring the inertia and the clamping force. These measurements are complemented by the measurements of temperature distributions using infrared cameras.

\section{Infrared camera set-up}

The IL-68 test rig was modified by installing the thermal imaging system. Remote temperature measurements by a thermal camera require a clear view of a measured surface. It was achieved by removing one of tested brake pads and making an opening there which serves as a measurement window. The modification of the test stand is shown in Figure 2.

(a)

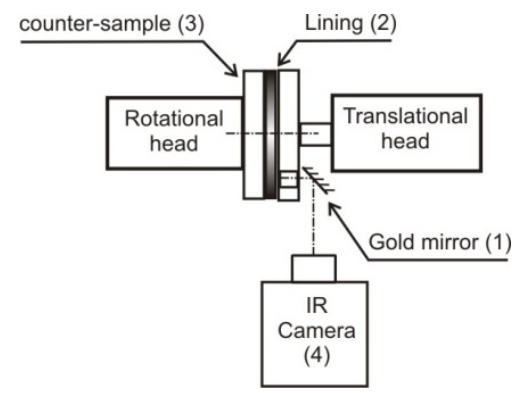

(b)

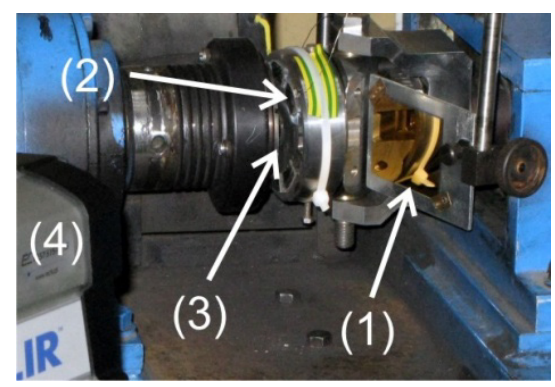

Figure 2: Additional thermal imaging system block diagram (a), real photo of a modified test rig (brake elements disconnected) (b).

An additional mirror (1) was applied due to the lack of free space to mount the thermal camera directly in front of a measurement window and to prevent the possible lens damage by brake pad debris. Due to the high rotational speeds and thus the rapid changes in temperature field, the infrared camera FLIR ThermaCAM SC6000 was used for testing $[8,9]$. Data were recorded in a reduced frame, matched to the dimensions of the measurement window. The camera allows us to write data at speeds up to 1000 of such reduced frames per second. Reduction of amount of data - i.e. area of measurement, was forced by the maximum data transfer rate in "Fast Speed" mode that can be reached for the camera used. Due to the high dynamics of the signal, measurements were carried out in the measuring range $200^{\circ} \mathrm{C}-750^{\circ} \mathrm{C}[8,7]$. Thus, in the initial phase of the test, in the absence of friction, and at the very end of the brake cooling, data from the infrared camera were not available. Spatial resolution proceed from IR 
camera lens focal length, distance from camera to sample, and detector pixel pitch is $0.1 \times 0.12 \mathrm{~mm}$.

\section{Numerical model of IL 68 stand}

FE numerical model of the system was developed, with a focus put on the frictional unit, described above. A more comprehensive description of the model can be found in [4]. Each of the 15 elements of the IL-68 was meshed using hexagonal elements (Figure 3, Figure 4).
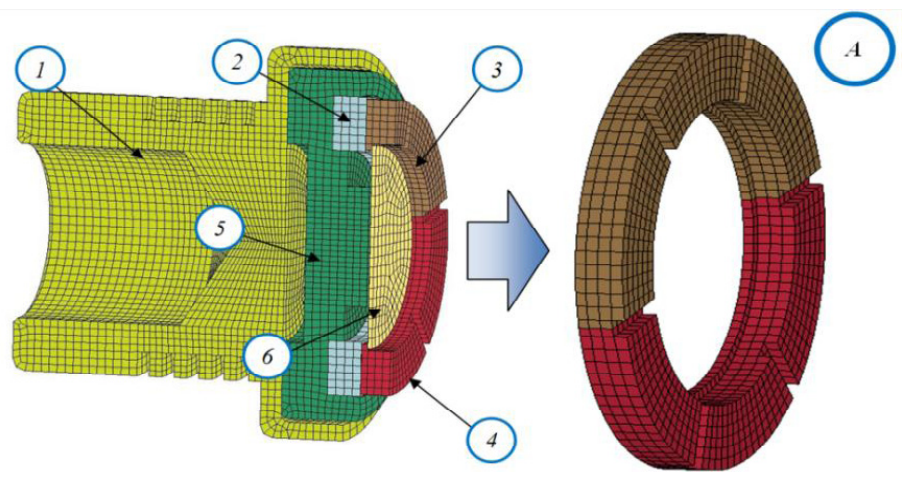

Figure 3: Numerical model of rotary head detailing the counter-samples: bushing counter-samples, base of the counter-samples (2), countersample $(3,4)$, counter-samples housing $(5)$, washer (6) [4].

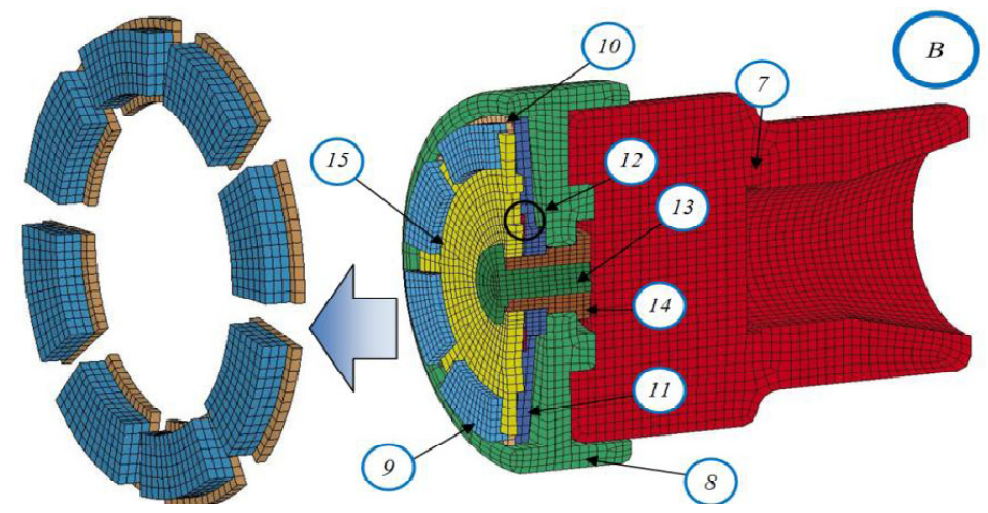

Figure 4: Numerical model of the translational head, detailing lining samples: samples housing (8), lining sample (9), base of the sample (10), rosette $(11)$, washer $(12)$, screws $(13,14)$, closing cap (15) $[5]$. 
Initial and boundary conditions have been defined in a manner that allowed us to kinematically force rotation of all components of the braked part (rotational head) and to prevent their sliding along the rotation axis (W1). Rotation of the rotational head was conducted by defining the angular velocity $\omega=628.31 \mathrm{rad} / \mathrm{s}$, with a vector lying on the axis X. In addition, density of the rotational head was increased in order to reflect the moment of inertia acting on the shaft of the device. Constrains applied to the pushing part only permit movement along the axis of the model and constraining movements in directions $\mathrm{Y}$ and $\mathrm{Z}$ (W2). Pressure realized by the hydraulic cylinder was imposed via force with a value estimated in experimental studies, i.e. $850 \mathrm{~N}$. The FE model, together with the adopted boundary conditions is shown in Figure 5.

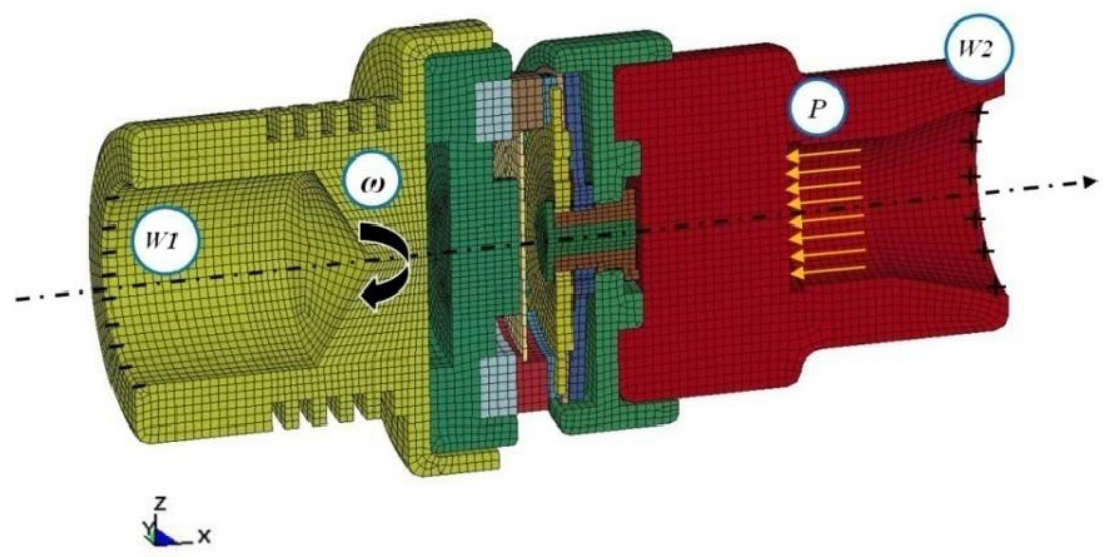

Figure 5: Boundary conditions applied to the FE model of stand.

Analyses were conducted with the assumption that both convection and radiation can be neglected, since only the first stage of braking process was covered by the analyses. In other words, the only source of heat in the model was friction and the whole work done by friction forces was converted into the thermal energy. See eqn. (1), where neglected terms are crossed out.

$$
F_{f} \frac{d S}{d t}=m c_{p} \frac{d T}{d t}+h A\left(T-T_{0}\right)+A \sigma T^{4}
$$

In the equation above: $\mathrm{F}_{\mathrm{f}}$ - friction force, $S$ - distance, $m$ - mass, $c_{p}$ - specific heat, $T$ - temperature, $h$ - heat transfer coeff., $T_{0^{-}}$ambient temperature, $t$ - time, $\sigma$-Stefan-Boltzman const.

Friction force $F_{f}$ was derived from well-known Coulomb model of friction with smoothing parameter stabilizing transition between static and dynamic friction. 
Contact normal force - a prerequisite for the friction force calculation - was obtained using the penalty function contact algorithm [5].

After several tests, the explicit integration scheme was chosen to solve system of equations. One the main reason of that choice was the fact that implicit methods were not noticably faster.

\section{Infrared measurements}

During remote temperature measurements the temperature distribution on the surface of a brake disc was recorded by a thermal camera. It was assumed, that the temperatures of brake disc and brake pads are the same. The temperature of brake disc increases during braking. The data from thermal images were processed using Altair software (FLIR Systems Inc.). Frame-by-frame analysis of the recorded thermal image sequences made it possible to find maximum temperature areas. The thermal images showing the beginning and the end (first frame after full stop) of braking cycle are presented in Figure 6.

(a)

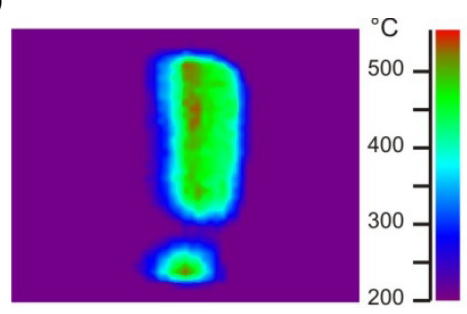

(b)

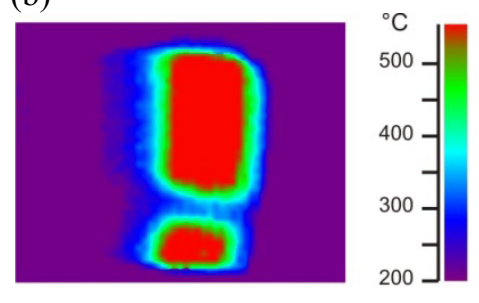

Figure 6: Thermal image of a brake disc initial braking phase (a), final image (full stop) (b).

Due to local imperfections of surface quality and mechanical alignment of disc-pad pairs the local high temperature points can be observed (Figure 6(a)). As the breaking continues the temperature distribution becomes more uniform cross the entire brake disc (Figure 6(b)). The temperature increase rate is very important information, as it determines the brake wear. The temperature was measured inside the rectangular area shown in Fig. 6(b). The measurement period (length of the recorded sequence) was produced from the infrared camera temperature range. For the first $300 \mathrm{~ms}$ the temperature is below $200^{\circ} \mathrm{C}$ and due to applied camera range there are no data available. The camera records the temperatures as high as $730^{\circ} \mathrm{C}$. This measurement range was chosen on the basis of previous, contact temperature measurements using thermocouples and multispectral pyrometers $[7,10]$. The plot showing the maximal temperature recorded on the surface of a brake disc during the initial phase of braking process is shown in Figure 7.

Temperature fluctuations results in the fact that consecutive frames show different areas of the tested brake disc. The actual size of the contact area between disc and pad determines the temperature value. The trend line was 


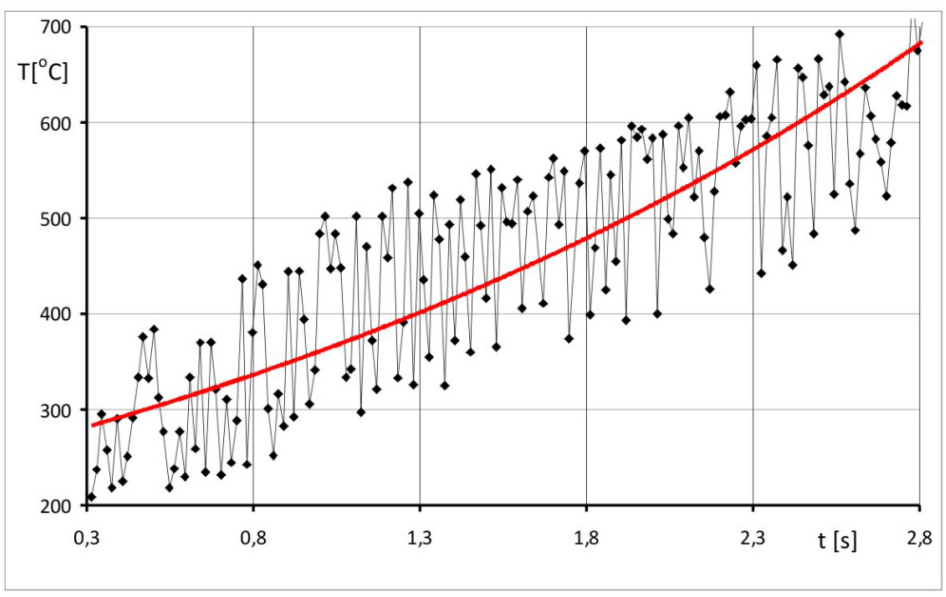

Figure 7: Maximal brake disc temperature values.

added to visualize the global temperature increase during braking. This line can be treated as heating characteristic for a given material $[8,9]$. In this case, after only 3 seconds the temperature exceeds $600^{\circ} \mathrm{C}$. Due to the applied camera and available choice of calibrated measurement ranges the further temperature increase could not been recorded. Nevertheless, the obtained information is important for the designers of braking systems. The occurrence of high temperatures requires high heat transfer out of the breaking system to maintain its functionality. It is particularly important in the case of special vehicles (e.g. ambulances) operating in the difficult conditions like mountain areas, where braking cycles are frequent and intense. In such cases poor heat dissipation leads to premature wearing of brake pads and discs.

\section{Numerical analysis results}

With a stable integration time step at the level of $5.5 \cdot 10^{-8} \mathrm{~s}$, it took about a week to cover the first stage of the braking process. In fact, the total CPU time to cover $2 \mathrm{~s}$ of analysis period was estimated at the level of 62,500 hours. It should be noted, that due to the nature of the simulated problem, simplification of the FE model was impossible. Figure 8 shows temperature distribution in the lining after 0.1 s. It can be seen that the "attacking" sides of pads are generating more heat than "trailing" sides, which is consistent with the observed behaviour on the test stand.

Figure 9 shows temperature and friction force change over the time, where temperature was converted from ${ }^{\circ} \mathrm{K}$ to ${ }^{\circ} \mathrm{C}$. Looking at the peak force values and temperature change for the same time moment, one can observe correlation between both quantities, as it was described by equation 1 .

The nature of changes in friction force values points to yet another difficulty associated with numerical analysis of the braking process - the level of discretization of contact surfaces. Very uneven and rapid change of the contact 


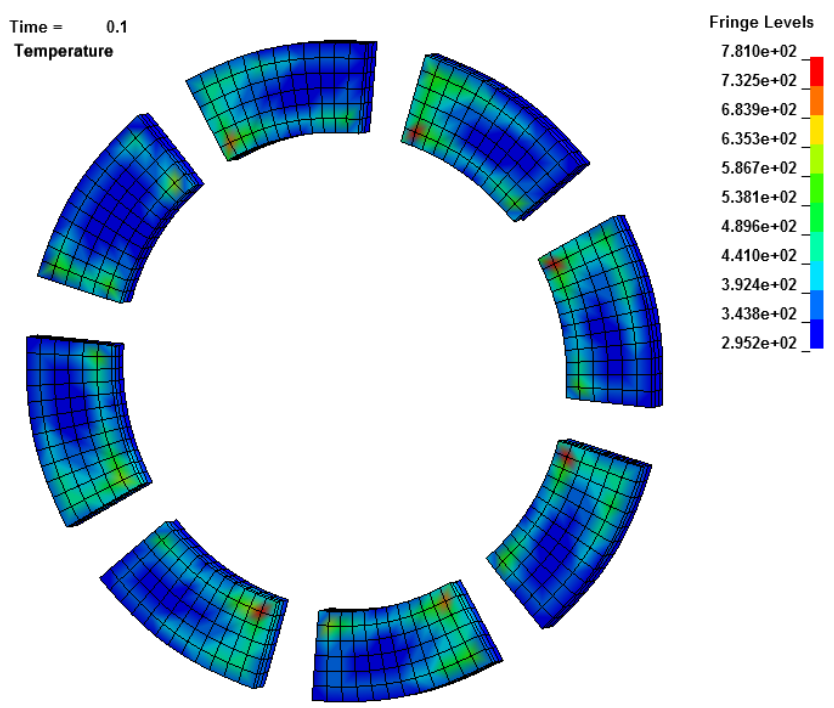

Figure 8: $\quad$ Temperature distribution on pads, at time $t=0.1 \mathrm{~s}$.

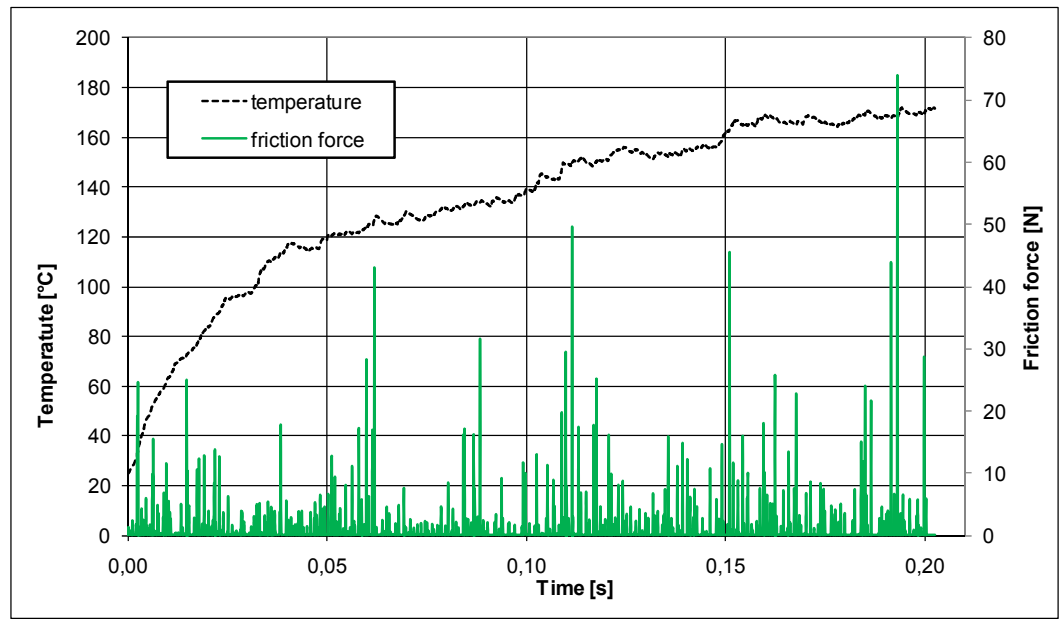

Figure 9: $\quad$ Temperature and friction force versus time.

force shows that the finite element mesh could have been too coarse. On the other hand, further decreasing of the element size would have resulted in further decreasing of time step making FE calculations unfeasible. One of the ideas of avoiding this problem is to use meshless methods, e.g. SPH approach. Another advantage of SPH usage is possibility to include in the model micro structural phenomena, such as wear due to local surface imperfection [6]. 
It should be noted that, despite all the difficulties mentioned above and lack of the overlap time range between FE results and measurements, temperature calculated using FE method approaches the values measured experimentally, achieving $170^{\circ} \mathrm{C}$ at $0.2 \mathrm{~s}$ (compared to $250^{\circ} \mathrm{C}$ at $0.3 \mathrm{~s}$ ). This indicates, that algorithm of thermomechanical coupling, as well as the assumed method of conversion of mechanical energy into the thermal energy, are working correctly.

\section{Conclusions}

Numerical simulation of laboratory tests proved that the application of FEMbased method is quite effective in simulating the transfer of kinetic energy into heat, which is the main energy transfer process during braking. In spite of the aforementioned obstacles (mainly time-consuming calculations and discretization requirements for the contact surfaces) which temporarily prevented the calculations to cover the whole braking cycle, thus making it impossible to directly compare the calculated and measured temperature data, it can be seen that the calculated temperature values (after the elongation of calculation period) will reach the level of measured ones. Currently conducted works are aimed at elongation of simulated brake process and implementation of SPH method for the modeling of brake pads operation. Additionally, on the basis of initial analyses, prediction of temperature values likely to occur in the braking system and the technical specifications of available thermal cameras it was concluded that the camera with the MCT detector type should be used for future experiments. Such cameras have broader measurement range, but lower thermal resolution than currently used camera with InSb detector. However, during measurements with high dynamic range of temperature changes the resolution of MCT-type camera is quite sufficient. Broadening of the measurement range will make it possible to compare the simulation and experimental results from the very beginning of brake process. Despite some problems with the saturation of camera detectors the thermal imaging proved its usefulness as a measurement technique for the recording of temperature values during brake examination.

\section{References}

[1] Uetz H., Föhl J., Wear as an energy transformation process, WEAR, pp. 253-264, 1978.

[2] Kennedy F. E., Thermal and thermomechanical effects in dry sliding, WEAR, 100, pp. 453-476, 1984.

[3] Suii N.P., Sin H.C., The genesis of friction WEAR 69, pp. 91-114, 1981.

[4] Baranowski P., Malachowski J., Mazurkiewicz L., Kajka R.: Budowa modelu numerycznego stanowiska badawczego okładzin ciernych IL-68, Górnictwo odkrywkowe, 3, 2010.

[5] Hallquist J., LS-DYNA Theory Manual, LST Co., 2005

[6] Baranowski P., Damaziak K., Malachowski J., Analysis of a brake including thermomechanical coupling, Proc. of the 19th Int. Conf. on Computer Methods in Mechanics, eds. A. Borkowski, T. Lewinski \& 
G. Dzierzanowski, Warsaw University of Technology: Warsaw, pp. 123124, 2011.

[7] Madura H., Kastek M., Piatkowski T., Automatic compensation of emissivity in three-wavelength pyrometers, Infrared Physics \& Technology, Volume 51, Issue 1, pp. 1-8, July 2007.

[8] Bielecki, Z., Chrzanowski, K., Matyszkiel, R., Piatkowski, T., Szulim, M., Infrared pyrometer for temperature measurement of objects of both wavelength- and time-dependent emissivity, Optica Applicata, Volume 29, Issue 3, , pp. 284-292, 1999.

[9] Piatkowski T., Polakowski H., Hots N., Examination of metallic surfaces for IR gray body sources, Proc. of the Int. $9^{\text {th }}$ Conf. Quantitative Infrared Thermography QIRT2008, Krakow, pp 185-190, 2008.

[10] Madura, H., Piatkowski, T., Powiada, E., Multispectral precise pyrometer for measurement of seawater surface temperature, Infrared Physics \& Technology, Vol. 46, Issue 1-2 SPEC. ISS, pp. 69-73, Dec. 2004. 